\title{
Discriminación laboral contra mujeres trabajadoras agrícolas del distrito de
} Sicaya, Huancayo Labor discrimination against agricultural women workers from the district of Sicaya, Huancayo

1 Licenciada en Trabajo Social, Magister en Políticas Sociales con mención en Promoción de la Infancia, Equilibrium Grupo de Servicios, anamanrique1951@hotmail.com, https://orcid.org/0000-0001-721 1-6545 2 Licenciada en Trabajo Social, Magister en Políticas Sociales con mención en Promoción de la Infancia, Equilibrium Grupo de Servicios, lucymg1407@gmail.com, https://orcid.org/0000-0001-5951-5445

3 Psicólogo por la Universidad de Carabobo de Venezuela, Equilibrium Grupo de Servicios, salvatoregullor@gmail.com, https://orcid.org/0000-0001-5951-5455 


\title{
Resumen
}

Históricamente, el mundo rural ha contado con un valioso apoyo: la mujer andina, quien contribuye a la economía familiar. Su participación en el mundo laboral, sin embargo, está sujeta a prácticas excluyentes y discriminatorias que vulneran sus derechos. El Valle del Mantaro es un contexto geográfico de actividad agrícola; en el distrito de Sicaya, se observa a decenas de mujeres, naturales de la zona o migrantes, dedicarse a labores agrícolas, y muchas de ellas son víctimas de dichas prácticas por parte del contratista y de sus propios compañeros de trabajo. En ese sentido, el objetivo de la investigación fue identificar cómo se presenta la discriminación laboral contra mujeres trabajadoras agrícolas del distrito de Sicaya, Huancayo (2017). Se llevó a cabo un estudio cualitativo de diseño etnográfico. Para la muestra, se seleccionó intencionadamente a 10 mujeres, solteras o no, con hijos, que laboran para un contratista y que son sujetos de prácticas excluyentes. A estas mujeres se les aplicó una entrevista en profundidad durante su tiempo de descanso; además, se observó su comportamiento durante las jornadas agrícolas y en el cuidado de sus menores hijos (observación participante). Se encontró que la preferencia del trabajo femenino o masculino depende del calendario agrícola; las mujeres madres reciben un trato intolerante del empleador, sobre todo cuando trabajan en compañía de sus menores hijos; las prácticas discriminatorias se expresan en la segregación por ocupación y sexo. Se concluye que la discriminación laboral por género contra la mujer se manifiesta en el trato despótico que reciben y en la diferenciación salarial respecto de los trabajadores varones.

Palabras clave: discriminación laboral, trabajo agrícola, peón agrícola, salario, trato.

\begin{abstract}
Historically, the rural world has counted on a valuable support: the Andean woman, who contributes to the family economy. However, her participation in the working world is subject to exclusionary and discriminatory practices that contravene her rights. The Mantaro Valley is a geographical context of agricultural activity; in the district of Sicaya, dozens of women, from this area or migrants, are engaged in agricultural work, and many of them are victims of such practices by the contractor and by their own work colleagues. In that regard, the research's objective was to identify how labor discrimination against agricultural women workers from the district of Sicaya, Huancayo, 2017 is. It was conducted a qualitative study of ethnographic design. For the sample, 10 women, single or not, with children, who work for a contractor and who are victims of exclusionary practices, were intentionally selected. It was applied an in-depth interview to those women during their break; their behavior was observed during their working time and in the care of their younger children (participant observation). It was found that the preference of female or male work depends on the agricultural calendar; women mothers receive intolerant treatment
\end{abstract}


from the employer, especially when they work in the company of their younger children; discriminatory practices are expressed in segregation by occupation and sex. It was concluded that labor discrimination against women takes the forms of a despotic treatment and salary distinctions.

Keywords: labor discrimination, agricultural work, agricultural worker, salary, treatment.

\section{Introducción}

La provincia de Huancayo, ciudad ubicada al pie de los Andes centrales del Perú, pertenece al Valle del Mantaro, conformado este por núcleos poblacionales con características urbanas y contextos rurales. Así, al lado noreste del Valle, se ubica la meseta de Sicaya, cuyos pobladores con experiencia rural se dedican a labores fundamentalmente agrícolas. En sus chacras trabajan varones y mujeres de acuerdo al calendario agrícola anual.

Una característica de las mujeres de las zonas rurales, tanto de América Latina como del Perú, es su multifuncionalidad de roles; se desempeñan como esposas y madres y, además, contribuyen a la economía familiar trabajando como peonas agrícolas. Se conoce que, en la sierra del Perú, la mujer trabajadora agrícola se desenvuelve en condiciones de desventaja, pues es víctima de prácticas laborales excluyentes y discriminatorias, con las cuales se comete una flagrante violación de sus derechos laborales, establecidos en normas internacionales de carácter vinculante.

Se analiza la importancia de la Convención de las Naciones Unidas sobre la eliminación de todas las formas de discriminación contra la mujer (Convención de la Mujer) en la promoción de los derechos de la mujer. Adoptada por la Asamblea General de la ONU en 1979, esta Convención ha sido ratificada por más de 170 naciones. Como un tratado contra la discriminación, tiene fortalezas y debilidades. Recientemente, se ha fortalecido mediante la introducción de un mecanismo de quejas individuales (el Protocolo Facultativo). (Fawole, 2008)

Históricamente, la mujer rural-campesina ha participado activamente en actividades agrícolas, sin embargo, en la actualidad, frente a la precariedad económica familiar, se ve obligada a trabajar a cambio de un salario. Así, Millar y Ridge (2009), en su estudio cualitativo longitudinal sobre madres solteras y sus hijos, refieren que, en un determinado momento, las madres dejaron el apoyo a los ingresos y comenzaron a trabajar durante al menos 16 horas por semana.

Se conoce poco aún sobre la participación laboral de la mujer acompañada de sus menores hijos; hecho que acentúa los procesos de segregación laboral por sexo y por su condición de madre. En un estudio longitudinal llevado a cabo en Nueva York, Lemer (1987) encontró, respecto a la participación en la fuerza laboral de madres con hijos pequeños y mediante un análisis de regresión múltiple, que las 
características de los niños, como la dificultad temperamental y la presencia de problemas físicos, eran tan potentes como las características demográficas para predecir las actividades del mercado laboral de la madre durante los primeros años de sus hijos.

En cuanto a la diferenciación salarial entre el varón y la mujer agrícolas, en la meseta de Sicaya, los empleadores pagan un monto inferior a la mujer respecto del que percibe el varón. Esta forma de discriminación, presentada en otros ámbitos laborales, ha sido abordada en numerosos estudios.

Destaca repetidamente la brecha entre los ingresos masculinos y femeninos en los sectores público y privado. Los autores abordan una manifestación pasada por alto de discriminación salarial contra las mujeres en el mercado laboral. Utilizando una encuesta de 384 oficiales principales de adquisiciones del sector público, analizan los efectos indirectos del género en el salario de las mujeres a través de la variable de autoridad que interviene. El género afecta la cantidad de autoridad que se delega a un empleado, lo que, a su vez, afecta la variación en el pago entre hombres y mujeres. (Alkadry y Tower, 2011)

El presente estudio tuvo como propósito conocer las prácticas excluyentes y sus manifestaciones: la discriminación laboral; la segregación ocupacional por sexo, condición de mujer y de madre trabajadora, y como estos procesos vulneran los derechos humanos, específicamente de la mujer rural. Existía la necesidad de percibir la problemática desde el punto de vista de las propias mujeres que viven esta realidad.

Conocer la discriminación laboral rural permite enriquecer el marco teórico de la especialidad de Trabajo Social sobre exclusión, discriminación, segregación en los espacios rurales, orientado al diseño de políticas de protección a la mujer peona agrícola; asimismo, posibilita perfilar la intervención del trabajador social en situaciones de exclusión social.

\section{Materiales y métodos}

El estudio cualitativo, de diseño etnográfico, considera a la etnografía como aquel método de investigación que busca describir a las personas, sus costumbres, su cultura; es un método relevante dentro de la investigación humanístico-interpretativa (Arnal del Rincón, 1992).

Entre los diseños de investigación, existe el etnográfico, utilizado "cuando se pretende describir, entender y explicar un sistema social" (Hernández Sampieri). Las técnicas de recolección de datos en el diseño etnográfico son la observación participante, en la que el observador participa de manera activa dentro del grupo estudiado, y la entrevista en profundidad, que es una interacción planificada entre dos personas y que obedece a un objetivo; en esta, el entrevistado da su opinión sobre un asunto y el entrevistador recoge e interpreta esa visión particular. 
Para la selección de los sujetos de estudio, se eligió intencionadamente a 10 mujeres rurales, solteras, madres, con hijos, que trabajan a diario en las chacras como peonas agrícolas a cambio de una remuneración y que son víctimas de prácticas discriminatorias ejercidas por el varón contratista.

Para el recojo de información, se ingresó a los predios agrícolas y se observó el comportamiento de las madres durante las jornadas laborales, el trato del contratista y las condiciones de trabajo (observación participante). Asimismo, se esperó la hora de descanso de las madres para abordarlas con la guía de entrevista y entablar conversación con ellas; tuvo que hacerse en varias sesiones por la naturaleza de las actividades que desarrollan.

\section{Resultados}

Tabla 1

Síntesis de resultados

\begin{tabular}{|c|c|}
\hline Referencias & Casos \\
\hline \multicolumn{2}{|l|}{ Menores hijos } \\
\hline Madres con hijos pequeños & 7 \\
\hline Madres que asisten al trabajo con sus hijos pequeños & 6 \\
\hline \multicolumn{2}{|c|}{ Trato del contratista con la mujer trabajadora que lleva a sus hijos } \\
\hline Trato tolerante & 2 \\
\hline Se incomoda & 4 \\
\hline \multicolumn{2}{|l|}{ Salario } \\
\hline Igual & 0 \\
\hline Diferenciado & 10 \\
\hline \multicolumn{2}{|l|}{ Monto } \\
\hline Mujer (35 a 45 soles) & 8 \\
\hline Varón (50 a 70 soles) & 8 \\
\hline \multicolumn{2}{|l|}{ Discriminación por ser mujer } \\
\hline Sí & 7 \\
\hline No & 3 \\
\hline
\end{tabular}


Discriminación por ser madre

\begin{tabular}{l|l}
\hline Sí & 7 \\
\hline No & 0
\end{tabular}

Discriminación laboral contra mujeres agrícolas: De las 10 informantes mujeres agrícolas, 7 tienen niños pequeños y 6 asisten al trabajo con ellos. 2 madres refieren que los contratistas son tolerantes ante la presencia de los niños y 4 afirman que se incomodan. Las 10 madres señalan que la remuneración diaria de la mujer con respecto a la del varón es diferenciada; 8 mujeres informan que perciben de 35 a 45 soles por jornada, en tanto el varón, de 50 a 70 soles.

3 de las entrevistadas sostienen que no son discriminadas por su condición de mujer; las 7 restantes dicen que sí.

Las 7 entrevistadas con hijos pequeños afirman que son discriminadas por su condición de madres.

Considerando que nuestra investigación es de carácter cualitativo, de diseño etnográfico, se consigna una única hipótesis. Para una mejor comprensión, se trabaja con tres ejes, cada uno de los cuales puntualiza conclusiones parciales orientadas a la conclusión final.

Eje 1: Discriminación de género expresada en la selección de la mano de obra femenina $y / o$ masculina

Tiempo que lleva como trabajadora agrícola: hace 18, 17, 14, 12, 10, 4 años que realizan esas actividades.

Dependiendo de la temporada, en correspondencia con el calendario agrícola anual, la peona accede con facilidad o no al trabajo:

El: Sí, en los meses de cosecha, se consigue más rápido...; E3: ...Consigo difícil, solo 2 a 3 veces por semana...; E8: ...No, antes era diferente... te contrataban por semana o mensual... ahora han migrado personas.

Preferencia por el trabajo agrícola femenino o masculino:

E3: En tiempo de inicio de siembra, prefieren contratar a los varones; ellos preparan la tierra, cargan el abono, porque ellos tienen más fuerza; E5: Dependiendo del trabajo que realizas, a veces llevan más a los hombres cuando es tiempo de preparación del terreno, y cuando es siembra, a las mujeres... 
Concluimos: la preferencia por el trabajo femenino o por el masculino depende del calendario agrícola-ganadero. Las actividades que el varón efectúa obedecen a su despliegue físico, fuerza, resistencia al frío. Las mujeres, en cambio, desarrollan actividades relacionadas con el manejo o destreza según temporada.

\section{Eje 2: Discriminación de género}

Trabajo diferenciado:

El: Cumplimos con el mismo horario, de 9 de la mañana a 5 de la tarde; hacemos diferentes trabajos, como por ejemplo en la cosecha de papa, zanahoria. Nosotras seleccionamos, pero en todo el varón carga toda la cosecha; E2: Yo trabajo igual, solo que los varones tienen más fuerza para los trabajos fuertes; E3: Yo trabajo menos, no igualamos al hombre en su fuerza; E4: El trabajo es igual; solo al momento de trasladar la carga para el camión, los hombres cargan los sacos; E5: Sí trabajo igual; la diferencia es que los hombres tienen fuerza y cargan los sacos de cosecha.

Aun considerando la capacidad física del varón, existen prácticas en el espacio rural donde se remarca los atributos físicos de este en desmedro de la condición física de la mujer.

Trato del contratista:

El empleador o contratista, dueño o no de los predios, es aquel que permanece con los trabajadores agrícolas (varón y mujer); existen reacciones diversas, que van desde la tolerancia al trato agresivo.

El: Hay patrones que te tratan bien, te dejan trabajar tranquilos, te ordenan de buena manera; como hay otros que son renegones, te gritan, te mandan hacer muchos trabajos extras; E2: Es bueno, nunca me ha tratado mal; además que hace 5 años solo voy a trabajar con un solo patrón; E3: Depende con qué contratista vayas; hay buenos, como también patrones que te gritan, te dicen "rápido", "¿no pueden trabajar más rápido?"; E4: Es malo, porque no me paga lo que me corresponde; otros me han demorado en pagar y me han dado en partes; también hay buenas personas, entienden y nunca te pagan menos; E5: Algunos son buenos, te dan para tu comida, coca; otros te hacen pasar horas de trabajo y no te quieren pagar más.

Esta situación se agudiza cuando la mujer madre trabajadora lleva a sus menores hijos consigo.

Trato del contratista a la mujer madre que labora con sus hijos:

El: Algunos contratistas se incomodan, porque no avanzamos el trabajo cuando lloran, pero mis compañeras de trabajo me apoyan mientras veo a mi hijo, ellas me ayudan a igualarme; E2: Hace 5 años que trabajo con un solo patrón y él ya conocía a mis niños y nunca se molesta, y al contrario, le invita sus frutas para mis niños; E3: 
Algunas contratistas se molestan porque cuando llora tengo que atenderlo, y también los mismos peones te dicen "para qué traes a tu hijo, no deja trabajar"; E4: En ocasiones se molesta porque hay momentos en el que tengo que atenderlo a él y las demás me llevan la delantera en el trabajo. E5: Siempre se molestan porque los chiquitos son molestosos y hacen mucha bulla, lloran cuando quieren dormir y eso les aburre a los jefes.

Concluimos: en los espacios rurales del Valle del Mantaro, las mujeres madres reciben un trato desmedido, intolerante, del empleador, sobre todo cuando trabajan en compañía de sus menores hijos.

Eje 3: La discriminación salarial

El trato desigual hacia las mujeres también se manifiesta en la remuneración que perciben.

Salario diferenciado por sexo:

El: No, las mujeres estamos con 40 o 45 soles por día; los varones ganan hasta 65 soles; E2: No, desde que empecé a trabajar, los varones han ganado más; E3: No, nosotras las mujeres por jornada recibimos 40 soles, y al varón le pagan 60 soles; E4: No, a mí me pagan algunas veces menos que otras mujeres, me dicen "no has avanzado por tu hijo", y a los varones les pagan más; E5: No, las mujeres ganamos hasta 45 soles el día de trabajo, y los varones ganan hasta 65 soles el día.

El varón gana más; la razón, responden nuestras informantes:

E1: ...porque el varón realiza el trabajo más fuerte...; E2: porque ellos cargan...; E4: ...solo por su fuerza...

Supeditado a su fuerza física, el empleador le proporciona al varón una paga mayor de la que percibe la mujer.

Concluimos: evidentemente, las prácticas excluyentes y discriminatorias son expresadas en la segregación por ocupación y por sexo.

\section{Discusión}

Las condiciones socioeconómicas de los hogares rurales, unidas a las prácticas culturales del mundo rural, propician la incorporación de la mujer del ande al trabajo agrícola. La participación femenina en estas actividades responde a un conjunto de creencias, visiones que el mundo rural recrea y enriquece, dando lugar a comportamientos en la vida laboral rural. Soportan nuestra apreciación Bielby y Baron (1986), para quienes, los empleadores, dadas sus creencias iniciales, podrían 
asignar a las mujeres tareas rutinarias en las que no se reconocen las habilidades reales de una mujer; en consecuencia, tales prácticas promueven la discriminación de género, expresada en la selección de mano de obra femenina y/o masculina.

E3: Hay veces en el que contratan a puras mujeres o también a puros varones, depende del trabajo que van a realizar.

Observamos que el empleador necesita de trabajadores varones y mujeres; no existe la preferencia por el trabajo femenino y/o masculino; durante el calendario agrícola anual, el empleador necesita de ambos.

Es necesario puntualizar que en el proceso del trabajo rural, el empleador asume posturas, acciones que reproducen desigualdades, ejerce actos diferenciados, atentando contra la igualdad de derechos debido a la discriminación de género. En la primera arista, se observa el trabajo diferenciado varón-mujer durante las jornadas agrícolas; es evidente la discriminación contra las mujeres rurales, indígenas, aun cuando el empleador reconoce que durante el calendario agrícola necesita de ambos. Al respecto, Hagen y Kahn (1975) sostienen que existen interacciones de competencias, cooperación.... La mujer peona agrícola percibe el comportamiento diferenciado del empleador; ellas enfatizan:

E1: Cumplimos con el mismo horario, de 9 de la mañana a 5 de la tarde... hacemos diferentes trabajos... pero en todo el varón carga toda la cosecha; E5: Sí trabajo igual, la diferencia es que los hombres tienen más fuerza.

En el distrito de Huamachuco, provincia de Sánchez Carrión, región La Libertad (2015), 30 mujeres denunciaron discriminación laboral por parte del teniente del distrito, Felipe Rojas, quien las llamó "inútiles" y precisó que necesitaba varones para el trabajo (Radio Programas del Perú, 2015). Sobre este particular, el modelo overcrowding alude a los procesos en los cuales, al trato del contratista, se enfatiza la idea en Cacho (2007): La triple discriminación: un breve bosquejo de la mujer indígena en Chiapas y en México, cuando la subordinación de género determina la forma de existencia en todos los ámbitos de su vida: en la familia, con la pareja, con los hijos e hijas, con la comunidad, con las autoridades, que imponen socialmente este modelo, además de controlar su cumplimento y exigibilidad.

Las visiones de la vida moderna reconocen que las mujeres tienen la capacidad de desenvolverse con el mismo éxito que los varones; sin embargo, en los espacios rurales, subsiste las miradas y comportamientos discriminatorios. 
El empleador que contrata mano de obra femenina agrícola es la siguiente arista de apreciación; en las interacciones con la mujer peona agrícola, muestra en muy pocos casos tolerancia y/o aceptación y el maltrato:

E4: Es malo, porque no me paga lo que me corresponde; otros me han demorado en pagar y me han dado en partes; también hay buenas personas entienden y nuca te pagan menos; E5: Algunos son buenos te dan para tu comida, coca, otros te hacen pasar horas de trabajo y no te quieren pagar más.

El enfoque de derechos sostiene que estos son inalienables según la Declaración Universal de Derechos Humanos, adoptada por la Asamblea General de la ONU; indica que se debe evitar la marginación, exclusión y discriminación. Para nuestro estudio, el comportamiento del empleador muestra un trato peyorativo, abusivo, desmedido; no se les paga oportunamente a las mujeres, se atenta contra su dignidad.

Otro aspecto del mismo tema es la arista referida al trato diferenciado, intencionado y selectivo contra la mujer trabajadora madre; aquella que se desplaza a las jornadas agrícolas acompañada de sus menores hijos.

E3: Algunas contratistas se molestan porque cuando llora tengo que atenderlo, y también los mismos peones te dicen "para qué traes a tu hijo; no deja trabajar"; E5: Siempre se molestan porque los chiquitos son molestosos y hacen mucha bulla; lloran cuando quieren dormir y eso les aburre a los jefes.

Millar y Ridge (2009) remarcan que la participación de las madres en actividades laborales es fundamental para el proyecto familiar. Se observa, sin embargo, que en los espacios agrícolas, no existen medidas de protección para la mujer madre que trabaja, que necesariamente asiste a la jornada agrícola con sus menores hijos. Reconocemos que existen programas como Cuna Más, por ejemplo, pero la mujer madre trabajadora debería acceder a otros servicios. Horbath y Gracia (2014) anotan que las diferencias existentes en la fuerza de trabajo masculino y las condiciones de la mujer, como ser o no jefa del hogar y el número de hijos, son fuente también de prácticas discrminatorias.

Y la última arista en este eje son los procesos de discriminación contra la mujer por su condición de ser mujer y madre; ellas perciben:

El: Yo siento que sí, porque cuando vas a buscar trabajo, prefieren llevar a las mujeres sin bebés; E4: Sí, como vengo con mis niños, los otros peones le dicen al patrón "para qué le llevas, sus niños van a molestar". 
La discriminación de género muestra dos vertientes: por ser mujer y por ser madre. La mujer madre carga con este estigma que, aunque tolerado y aceptado por la sociedad rural, contraviene sus derechos.

El diario Perú 21 (2014) reporta que las mujeres consideran que se les excluye del mercado laboral en el país. Por otra parte, Cacho (2007) remarca, en La triple discriminación: un breve bosquejo de la mujer indígena en Chiapas y México, la subordinación de las mujeres indígenas desde la perspectiva de una triple opresión: su clase, su género y su condición étnica; así, las mujeres indígenas son oprimidas y excluidas por ser pobres, por ser mujeres y por ser indígenas. En nuestro contexto, a partir de este estudio, se añadiría una razón más de exclusión de la mujer indígena: llevar consigo a sus hijos a las zonas de trabajo.

Concluimos: la mujer rural que se desenvuelve como peona agrícola es sujeto de diversas formas de discriminación de género reflejada en el trabajo diferenciado varón-mujer, porque no tiene la capacidad física de este, y en el trato peyorativo por parte del contratista, especialmente por su condición de madre.

La discriminación salarial es la expresión del trato diferenciado contra la mujer que trabaja:

El: No, las mujeres estamos con 40 o 45 soles por día; los varones ganan hasta 65 soles; E5: No, las mujeres ganamos hasta 45 soles el día de trabajo y los varones ganan hasta 65 soles el día.

El Comercio (2017) señala las prácticas más comunes de discriminación laboral femenina; precisa que en Colombia, las trabajadoras femeninas llegan a percibir en promedio un sueldo $25 \%$ menor al de sus colegas varones. Se aprecia, además, que según un estudio de la Universidad Complutense de Madrid, los varones que ingresan a laborar en trabajos "pensados para ellas" suelen recibir un buen salario; contrariamente, si las mujeres hacen lo mismo, reciben pagos realmente bajos.

La teoría del gusto por la discriminación, o teoría de Becker, sostiene que históricamente las personas que han sufrido mayor discriminación son las mujeres y las personas de color. Existen procesos selectivos intencionados, como la segregación ocupacional o laboral por sexo; así, los hombres reciben salarios más altos aunque desarrollen las mismas funciones que las mujeres.

Dex (2009) refiere que la teoría dual del mercado laboral explica estas diferencias en el salario. Concluimos: existen construcciones culturales manifestadas en formas de pensar y en actitudes; sin mencionarlo, se acepta que el hombre es, por naturaleza, superior a la mujer. En consecuencia, cuando la mujer trabaja, se denotan las 
prácticas diferenciadas, el desprecio y un trato inferior. Estos comportamientos son aceptados y tolerados por la sociedad rural en el Perú, como en el Valle del Mantaro, donde se ubica la meseta de Sicaya.

\section{Agradecimiento}

A las mujeres del distrito de Sicaya (provincia de Huancayo) que realizan trabajos agrícolas, las mismas que brindaron la información para la ejecución de esta investigación.

\section{Referencias bibliográficas}

Alkadry, M. G., y Tower, L. E. (2011). Covert Pay Discrimination: How Authority Predicts Pay Differences between Women and Men. Public Administration Review, 71 (5), 740750.

Becker, G. (1971). The economics of discrmination. Chicago, Estados Unidos: University of Chicago Press.

Bielby, W. T., y Baron, J. N. (1986). Men and Women at Work: Sex Segregation and Statistical Discrimination. American Journal of Sociology, 91 (4), 759-799.

Cacho, N. (2007). La triple discriminación: un breve bosquejo de la mujer indígena en Chiapas y en México. Recuperado de http://www.biodiversidadla.org/Documentos/La-triple-discriminacion-un-brevebosquejo-de-la-mujer-indigena-en-Chiapas-y-en-Mexico

El Comercio. (7 de febrero de 2017). Discriminación laboral femenina: las 4 prácticas más comunes. Recuperado de https://elcomercio.pe/viu/actitud-viu/discriminacionlaboral-femenina-4-practicas-comunes-163242-noticia/

Fawole, O. I. (2008). Economic violence to women and girls: Is it receiving the necessary attention? Trauma, Violence \& Abuse, 9(3), 167-177.

Hagen, R. L., y Kahn, A. (1975). Discrimination Against Competent Women. Journal of Applied Social Psycholoy, 5(4), 362-376.

Horbath, J., y Gracia, A. (2014). Discriminación laboral y vulnerabilidad de las mujeres frente a la crisis mundial en México. Economía, Sociedad y Territorio, 14(45). Recuperado de http://www.scielo.org.mx/scielo.php?script=sci_arttext\&pid=\$140584212014000200006 
Millar, J., y Ridge, T. (2009). Relationships of care: Working lone mothers, their children and employment sustainability. Journal of Social Policy, 38(1), 103-121.

Perú 21. (5 de marzo de 2014). Discriminación laboral: el 88\% de mujeres considera que se les excluye. Recuperado de https://peru21.pe/economia/discriminacionlaboral-88-mujeres-considera-les-excluye-147677-noticia/

Radio Programas del Perú. (6 de enero de 2015). Huamachuco: 30 mujeres denuncian discriminación laboral. Recuperado de https://rpp.pe/peru/actualidad/huamachuco-30-mujeres-denunciandiscriminacion-laboral-noticia-757188 